\title{
Modeling and Structural Analysis of Rear Axle Casing of Tractor
}

\section{E.Mahavishnu', R.Manikandan ${ }^{2}$, M.Ramakrishna ${ }^{3}$}

1, 2,3 Assistant Professor, Department of Aeronautical Engineering, Bharath Institute of Higher Education and Research, Chennai.

Article History: Received: 11 January 2021; Accepted: 27 February 2021; Published online: 5 April 2021

ABSTRACT: In this research paper we are going to analysis the rear axle hosing of the tractor for circular, rectangular and elliptic shape to improving the structural stability and load carrying capacity, deformation and fatigue analyze done in FEA methodology, model designed in CATIA software.

Keywords: Rear axle hosing, FEA, Rectangular and elliptical hosing, CATIA

\section{INTRODUCTION}

In tractor rear axle having more amount dynamic load because continuous rotation, here the applying load and cycle of rotation also high, therefore the axle component life will be reduce or it will be damaged before reaching maximum load factor. The rear axle material should withstand this fatigue load that's why choosing SG 500 casting iron; it has more wear resistance, high toughness, fatigue resistance and also having excellent ductility characteristic.

Here we going to modify the rear Axle shape from circular to rectangular and elliptical shape but the material are same. With help of Finite element method technique we can able to ductile and fatigue analysis this modified shape for required boundary condition,

The model is designed in CATIA software, meshing and analysis done in FEA software, finally we compare the deformation fatigue results for three shape.

\section{MODELING}

Modeling of rear axle housing done in CATIA Software for TAFE 1002 4WD tractor the model have the circular shape of the housing structures, these structures can be modified as the rectangular shape and elliptical shapes

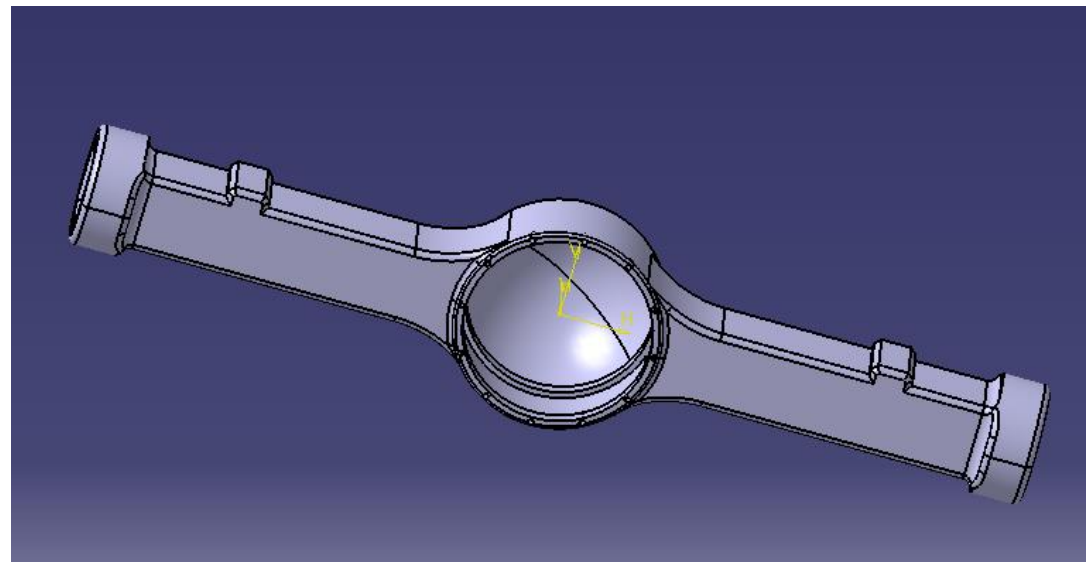

MODELING OF REAR AXLE CASING FOR TRACTOR

Structural Boundary Conditions for rear axle housings

Support: fixed support

Load: $1000 \mathrm{~N}$

Length: $1 \mathrm{~m}$

No of division: 1000 
FEA model

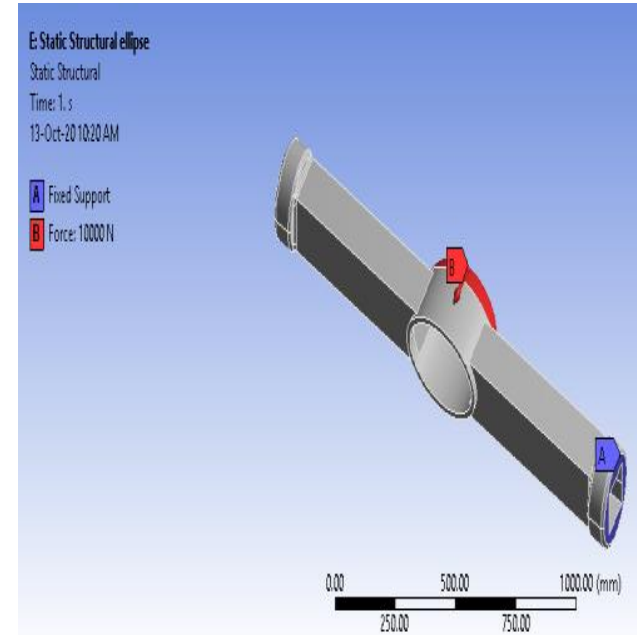

Circular shape
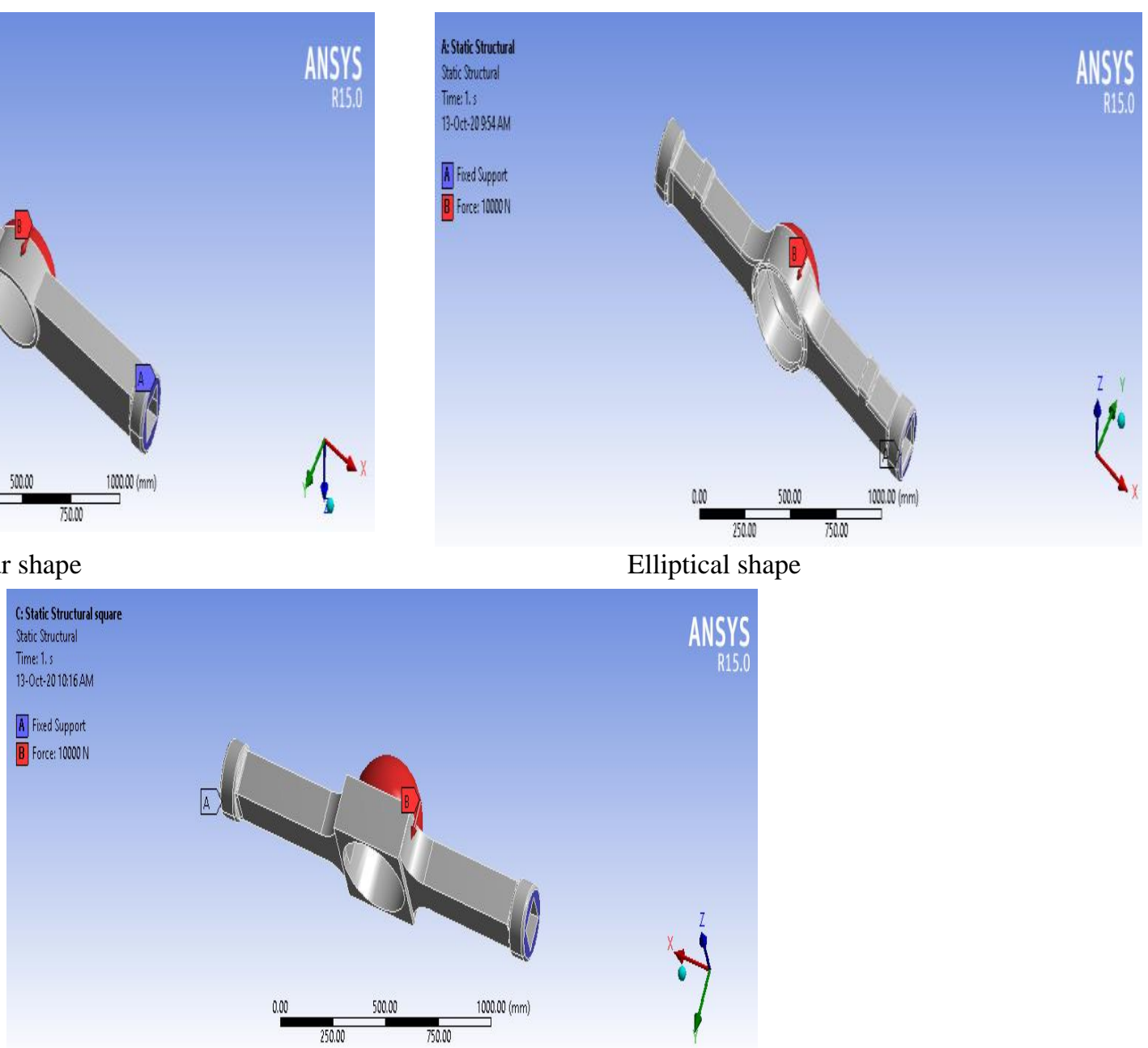

Rectangular model

\section{Meshing:}

Meshing done in FEA software here we used tetrahedral elements for more accuracy.

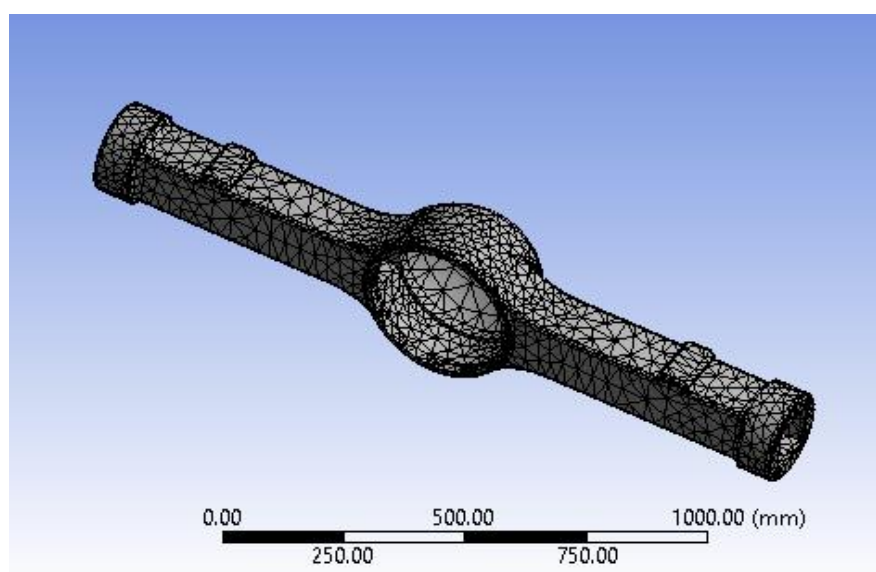

Circular shape

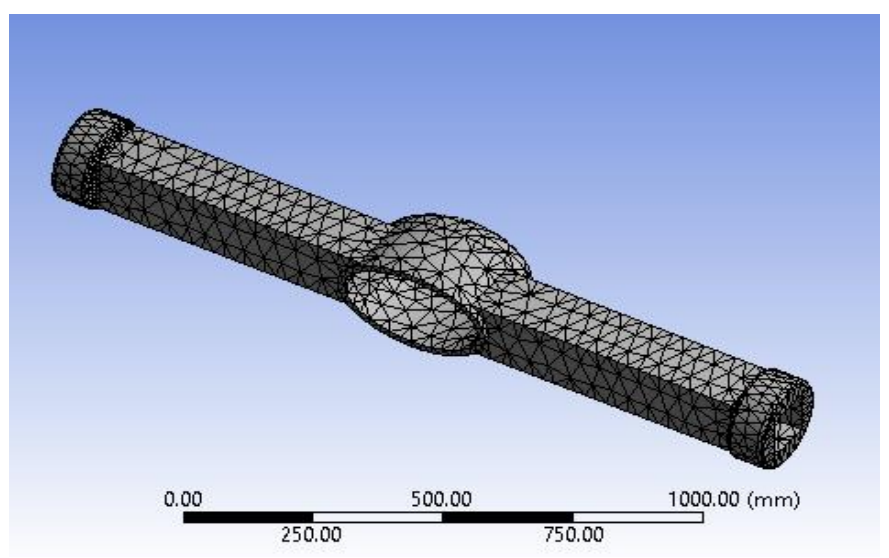

Elliptical shape 
Structural Analysis and Results:

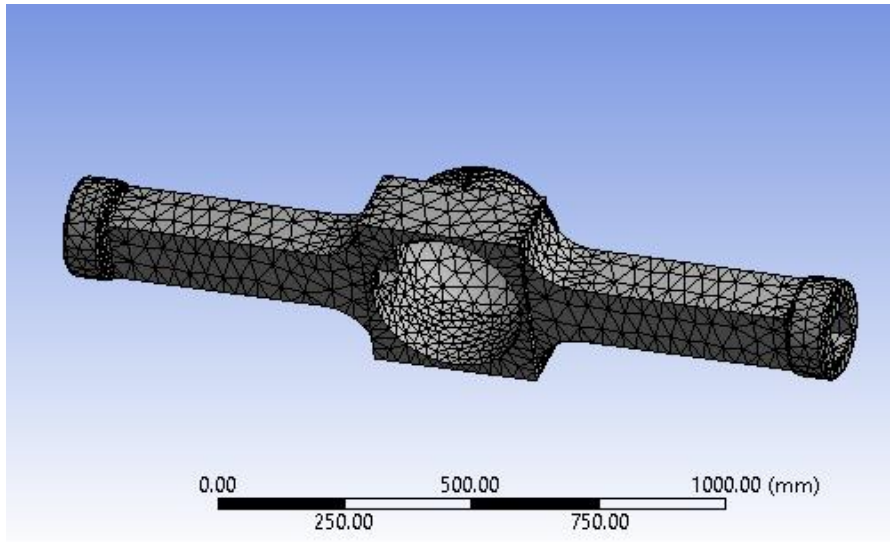

Rectangular shape
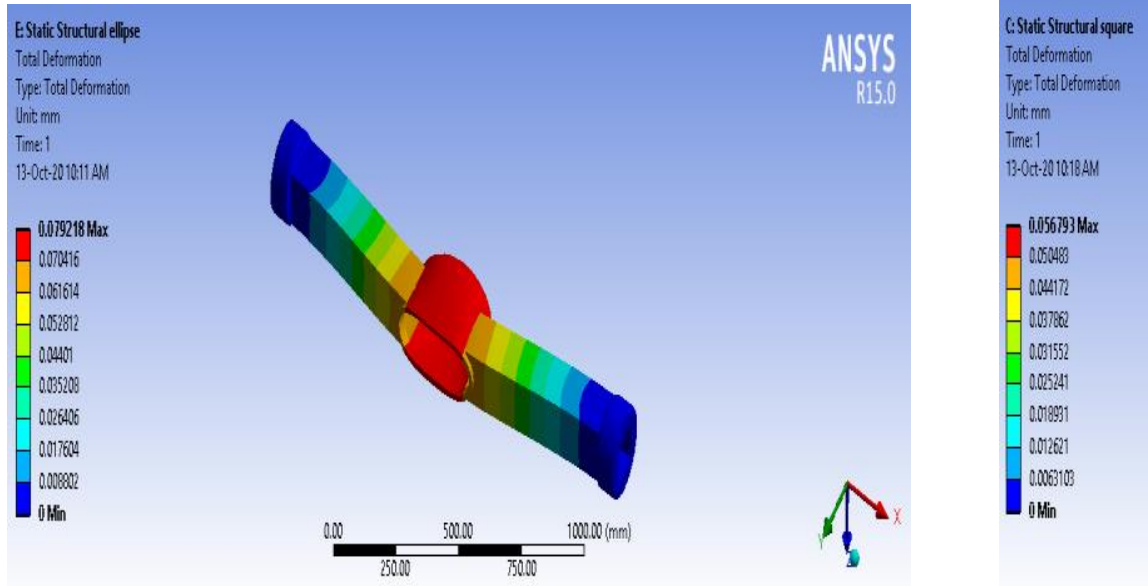

Elliptical shape

Rectangular shape

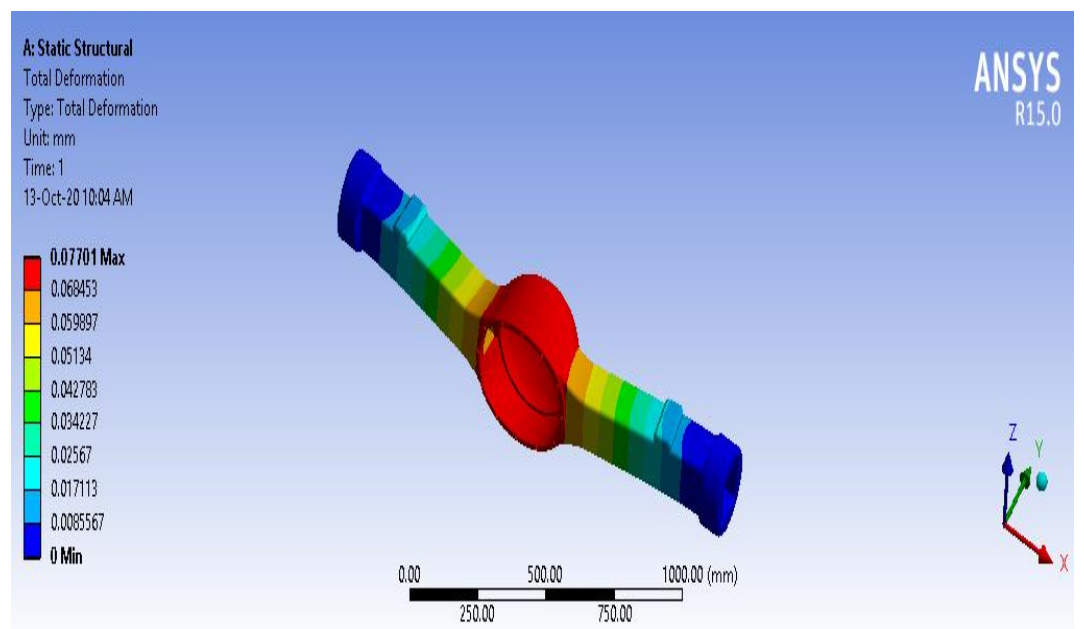

Deformation results: 


\begin{tabular}{|c|c|}
\hline Shape & Total deformation \\
\hline Circular shape & 0.07701 \\
\hline Ellipse shape & 0.079218 \\
\hline Rectangular shape & 0.056793 \\
\hline
\end{tabular}

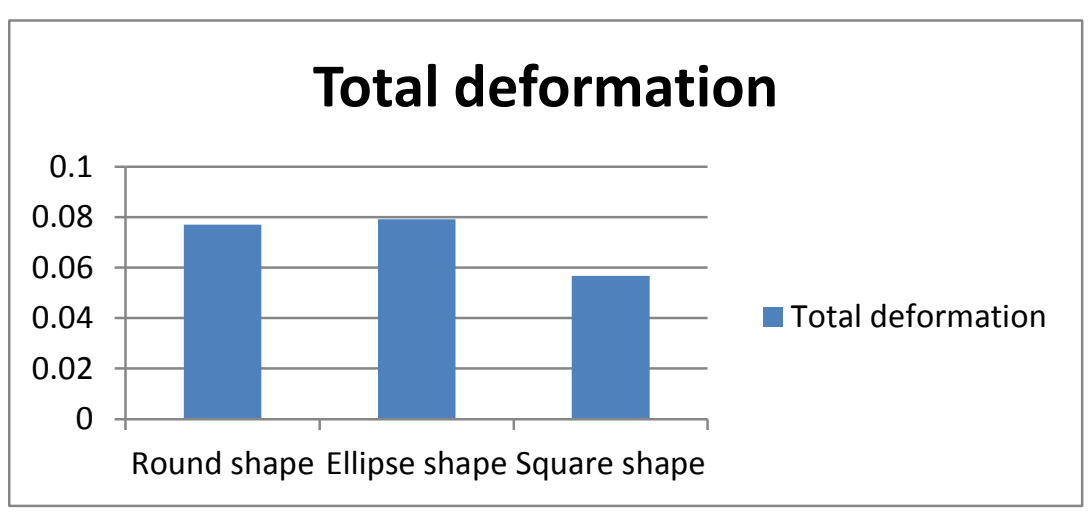

Total Deformation results of rear axle housings

\section{EQUIVALENT STRESS}

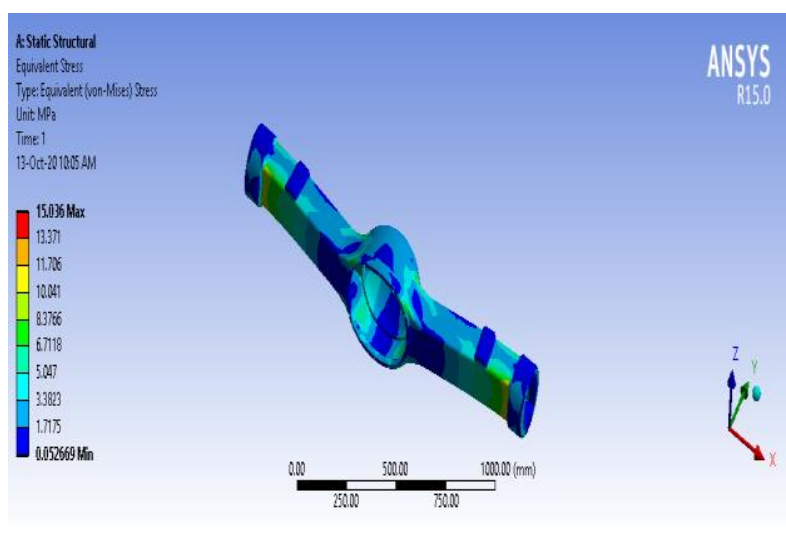

Elliptical Shape

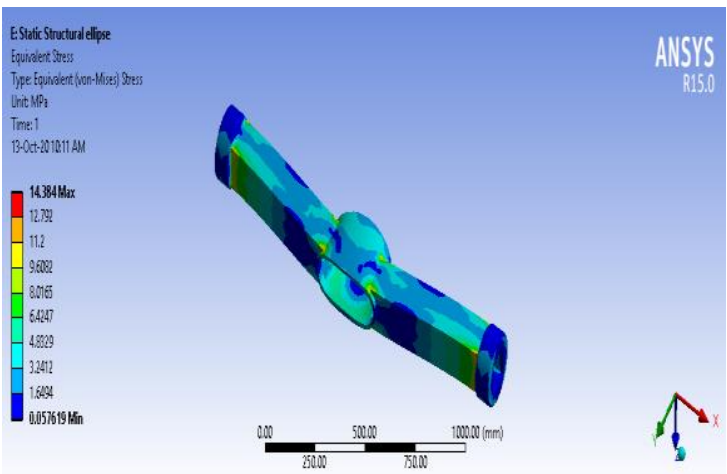

Circular shape

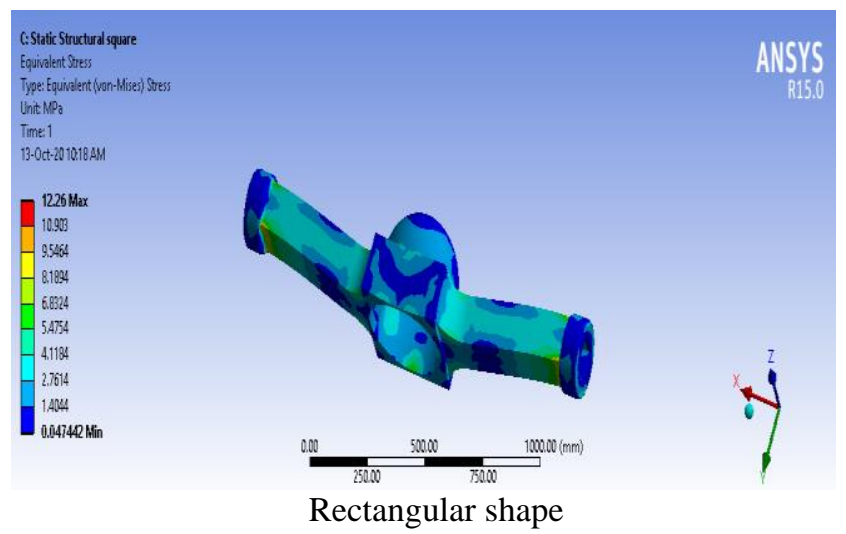


Equivalent stress results

\begin{tabular}{|c|c|}
\hline Shape & Equivalent stress \\
\hline Circular shape & 15.036 \\
\hline Ellipse shape & 14.384 \\
\hline Rectangular shape & 12.26 \\
\hline
\end{tabular}

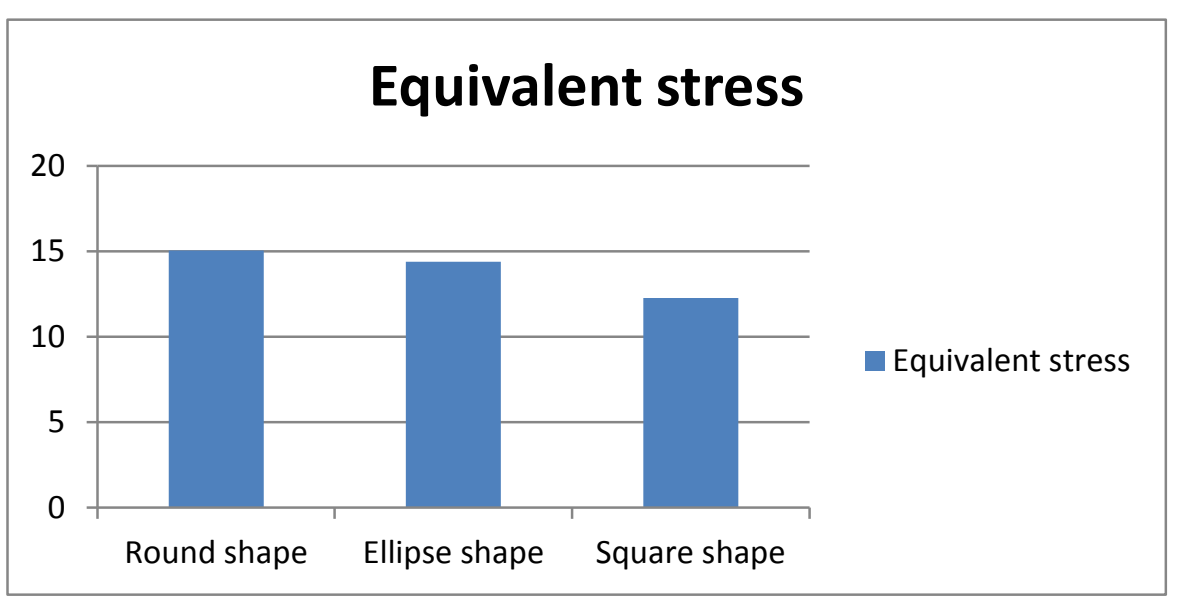

\section{MAXIMUM PRINCIPLE STRESS}

Circular shape

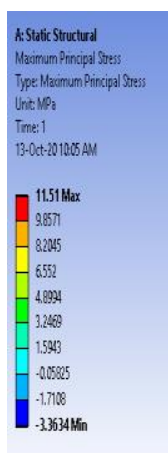

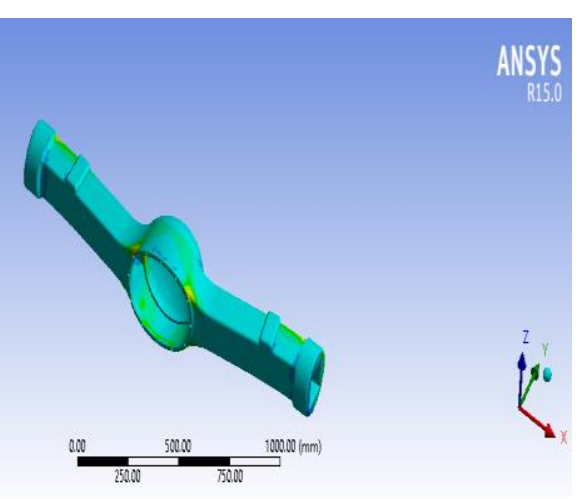

\section{Ellipse shape}

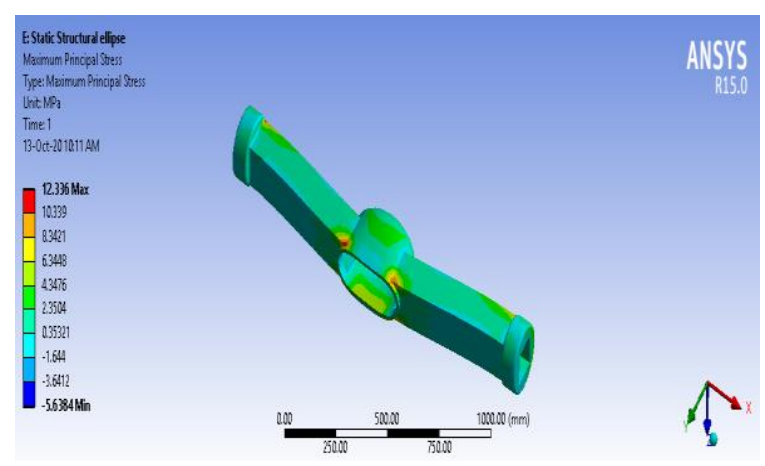

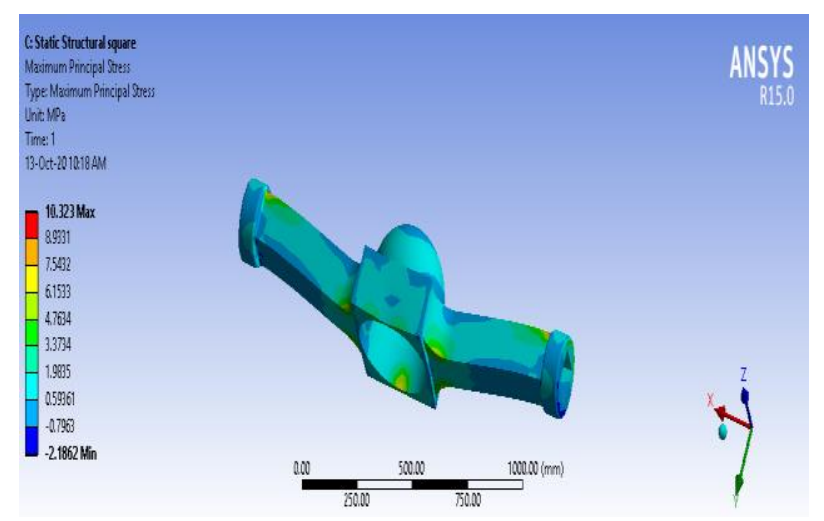




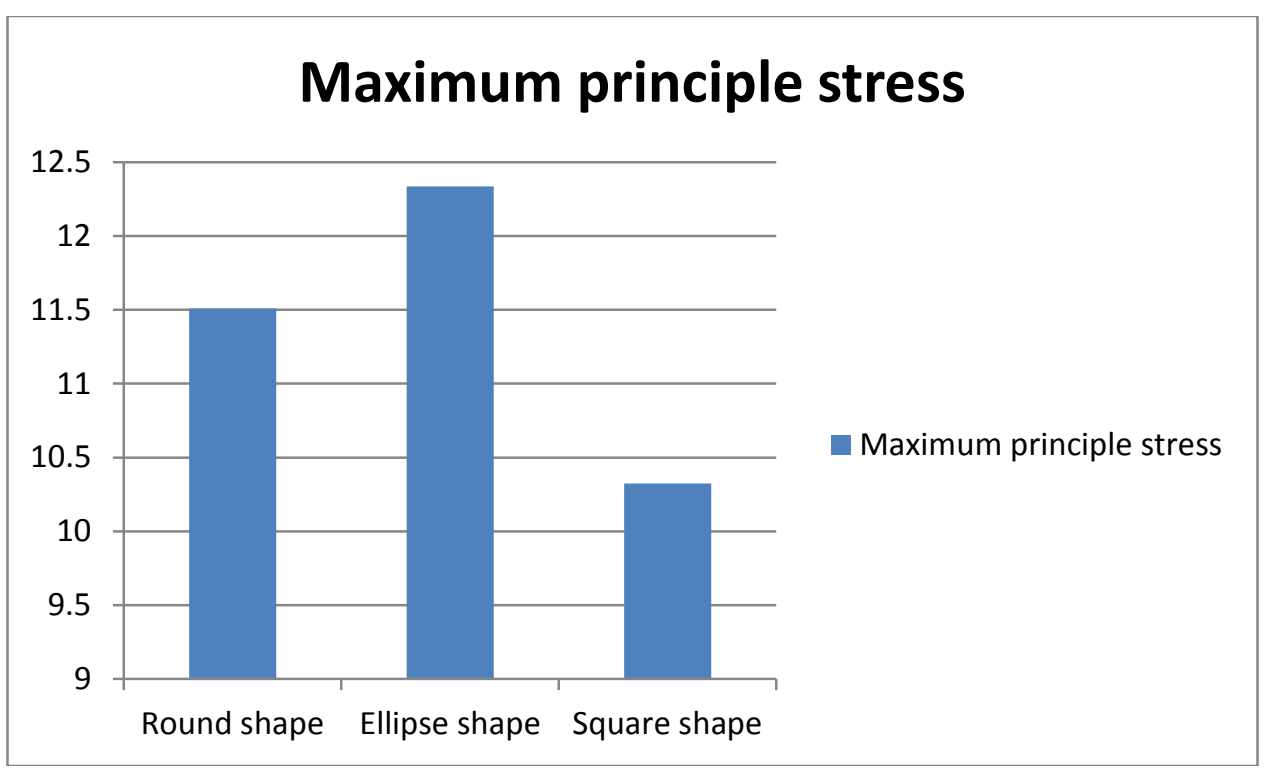

\begin{tabular}{|c|c|}
\hline & Maximum principle stress \\
\hline Circular shape & 11.51 \\
\hline Ellipse shape & 12.336 \\
\hline Rectangular shape & 10.323 \\
\hline
\end{tabular}

MODAL ANALYSIS

Frequency 1
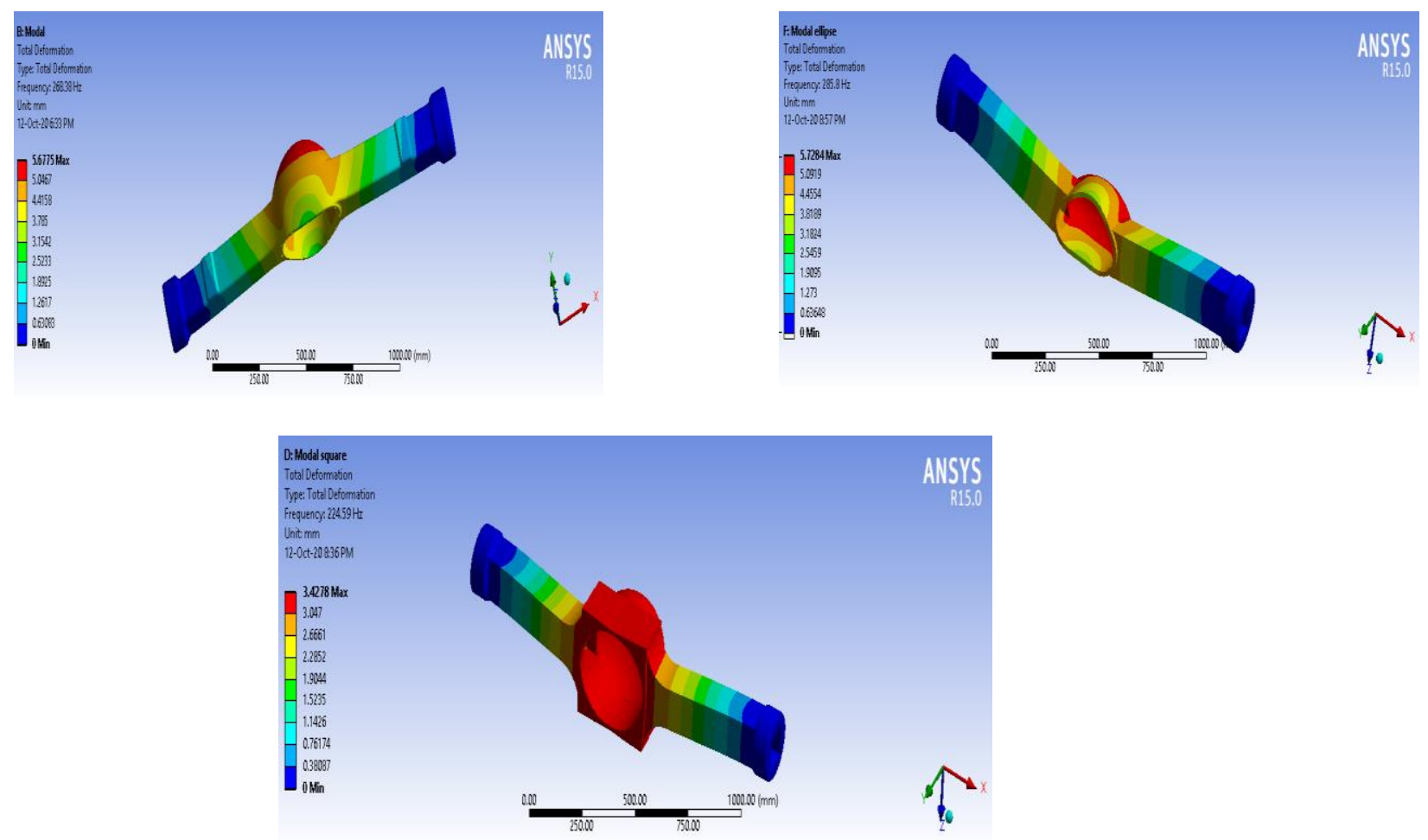

Rectangular shape 
Frequency 2

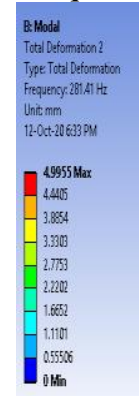

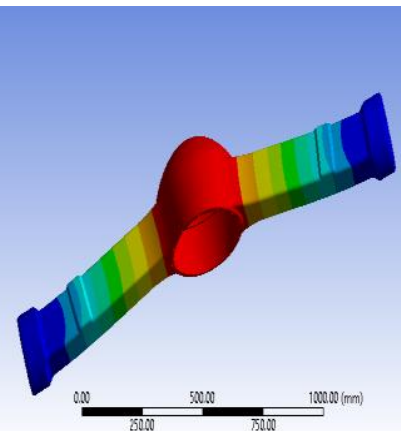

Circular shape

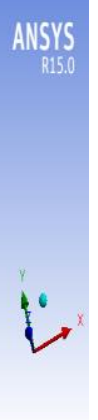

D: Model square
Total Deformation?

Total Detomatoon?

frequency; $240.12 \mathrm{~Hz}$

Uhitm

12.0ct-20637 P

48021 Hax

-4.205
-3.755
-3.54

$-3.2014$

-2.678
-2.134

$-1.6007$

-1.067
-0.53557

\section{Frequency 3}

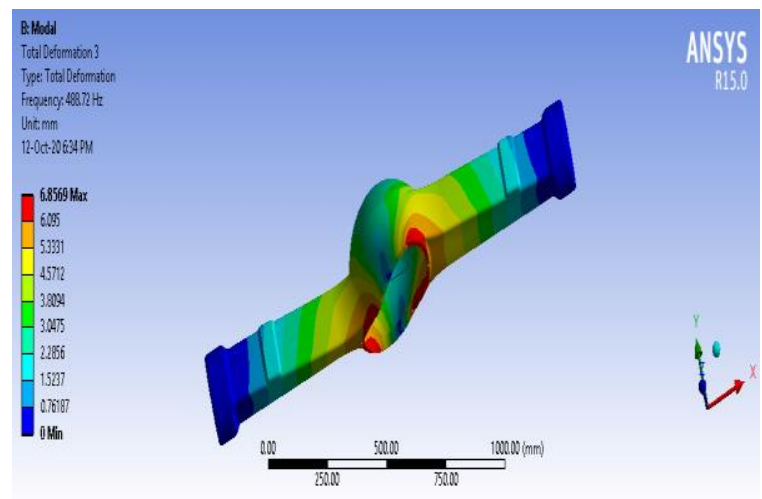

Circular shape

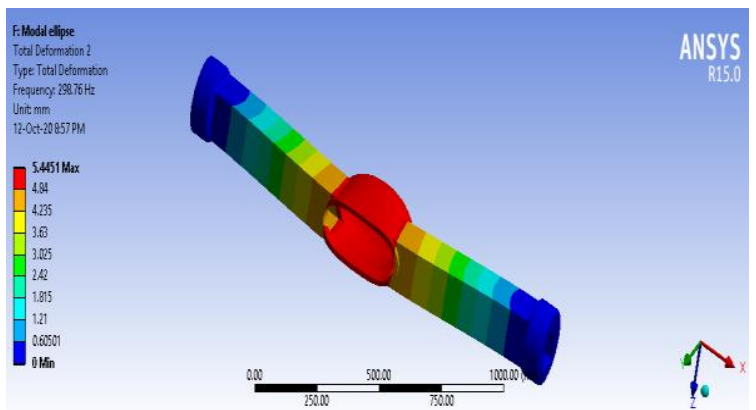

Ellipse shape

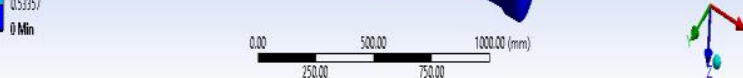

Rectangular shape

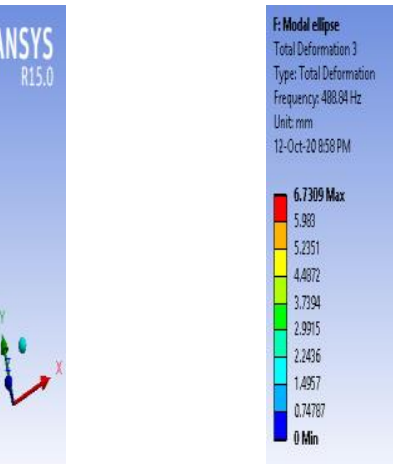

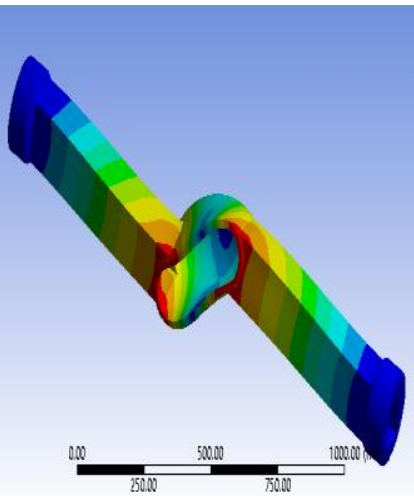

Ellipse shape
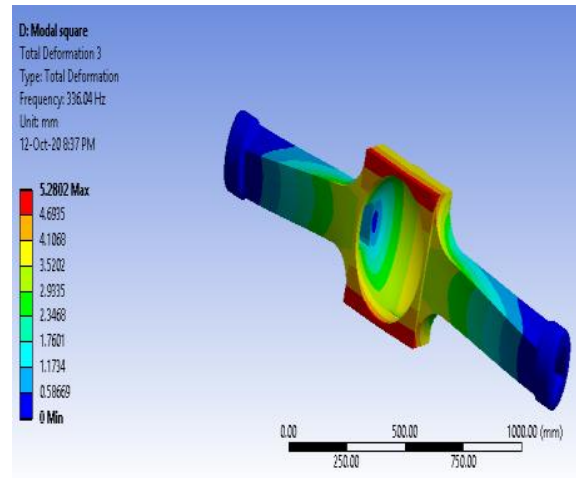

Rectangular shape 
Frequency 4
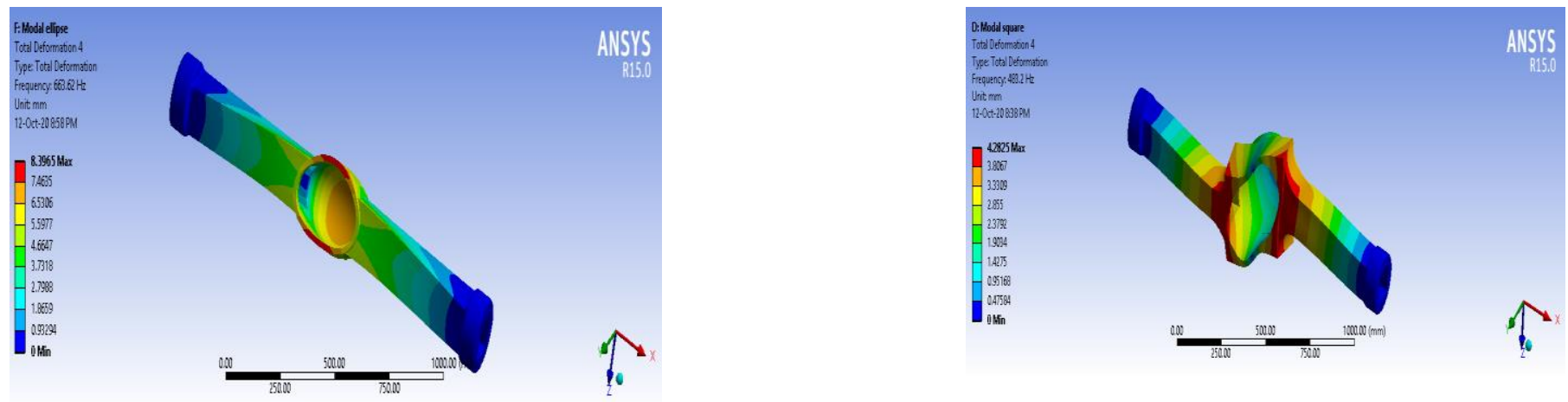

Ellipse shape

Rectangular shape

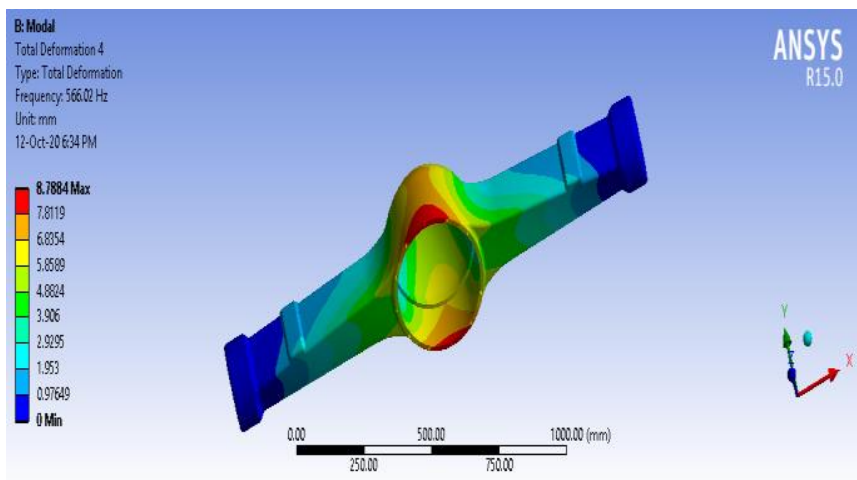

Frequency 5

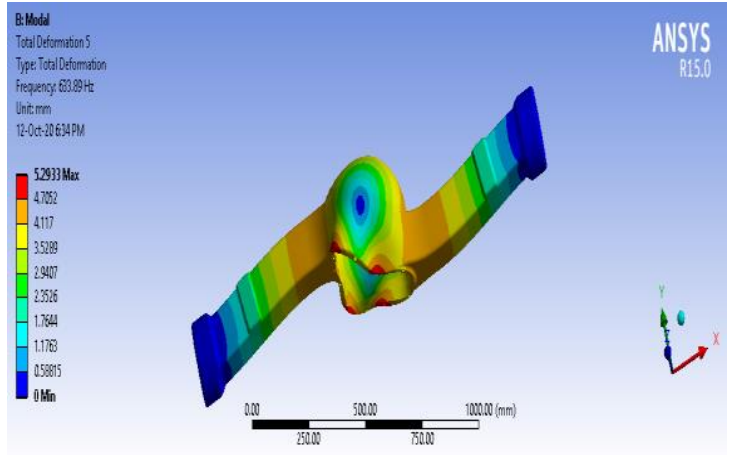

Circular shape

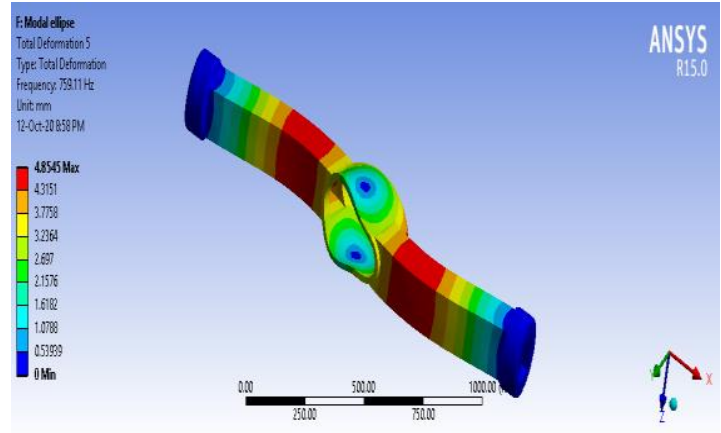

Ellipse shape

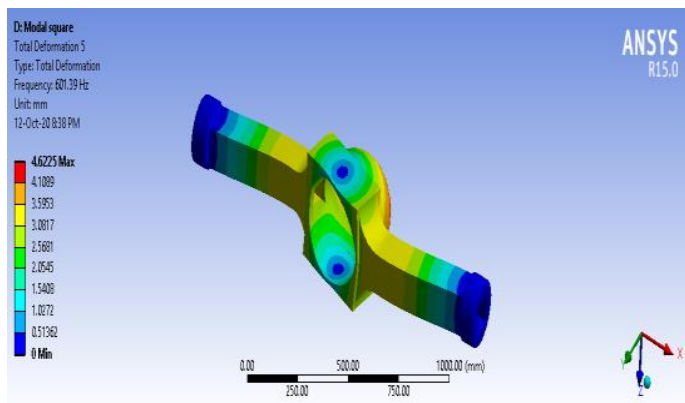

Rectangular shape 
Frequency 6

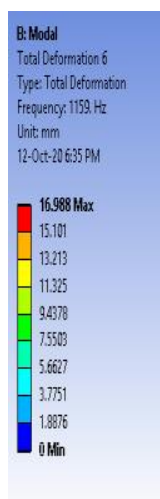

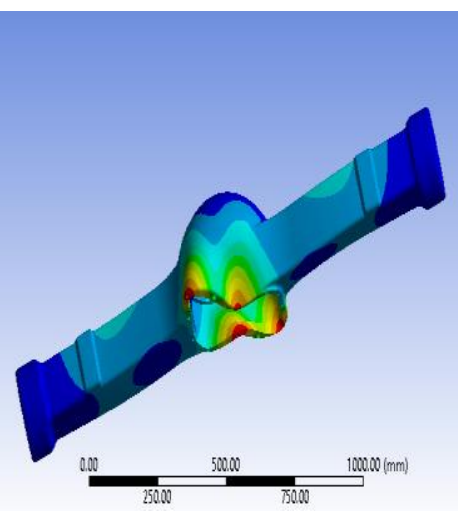

Circular shape

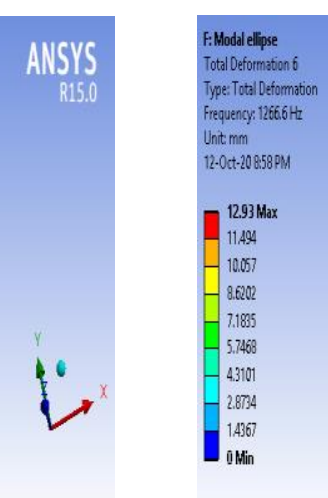

Ellipse shape
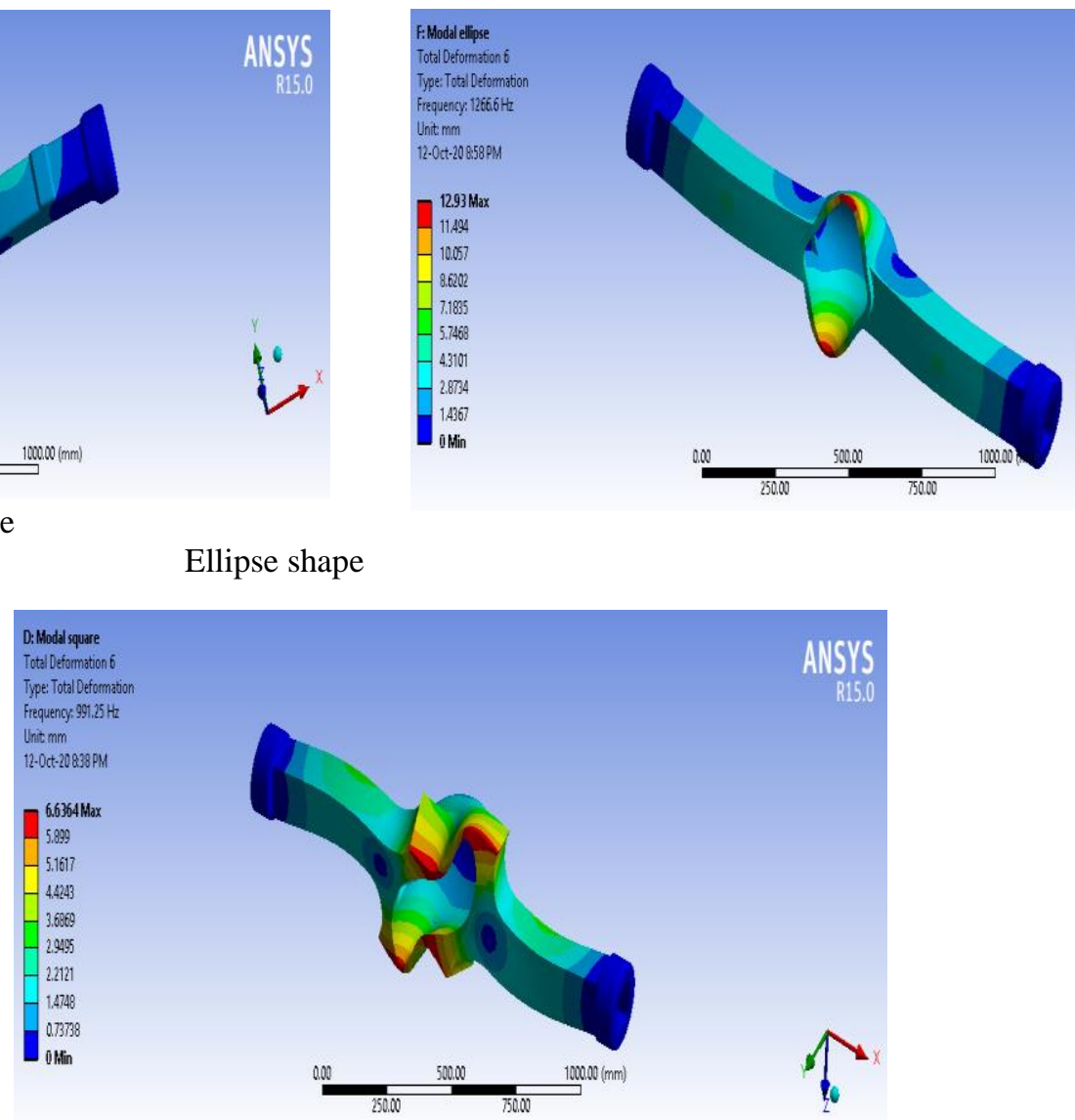

Rectangular shape

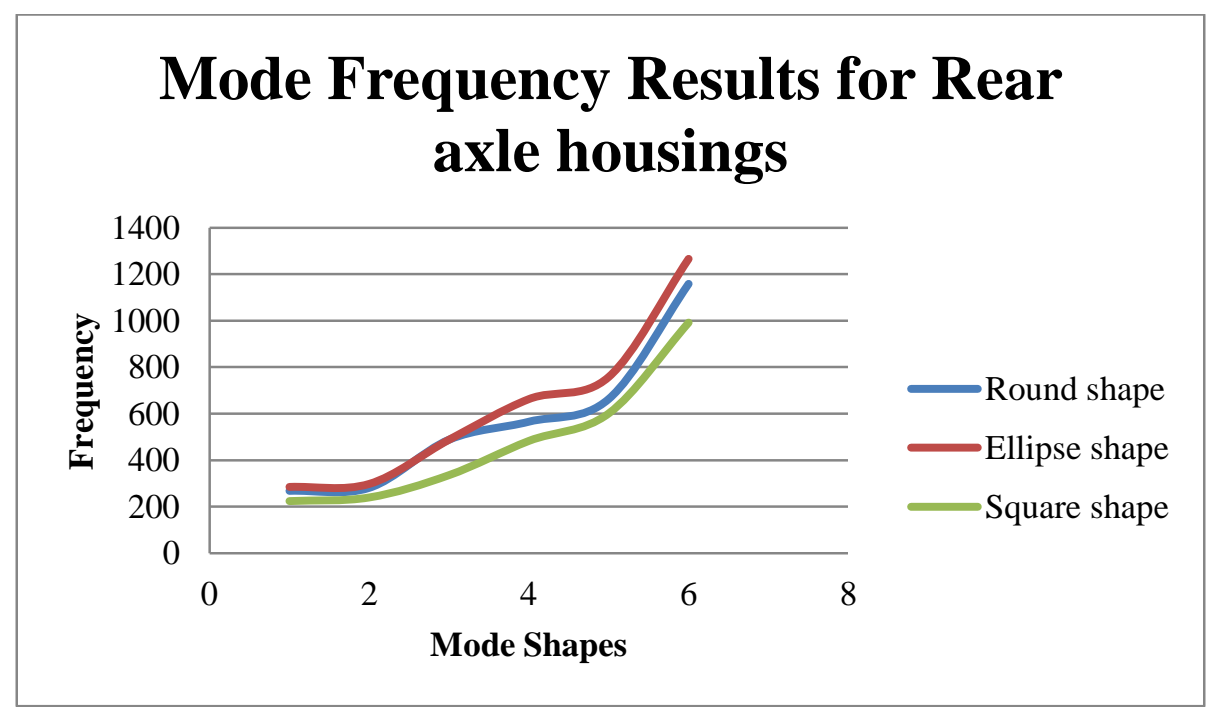




\begin{tabular}{|c|c|c|c|}
\hline & Round shape & Ellipse shape & Square shape \\
\hline Frequency 1 & 268.38 & 285.8 & 224.59 \\
\hline Frequency 2 & 281.41 & 298.76 & 240.12 \\
\hline Frequency 3 & 488.72 & 488.84 & 336.04 \\
\hline Frequency 4 & 566.02 & 663.62 & 483.2 \\
\hline Frequency 5 & 663.89 & 759.11 & 601.39 \\
\hline Frequency 6 & 1159 & 1266.6 & 991.25 \\
\hline
\end{tabular}

\section{Conclusion}

From the results of rear axle housing structural and modal analysis of tractor axle with the various housing shapes like circular, ellipse shape and square shape of the housings, deformation of the housings rectangular shape got very less stress and principle stress also got very less in the applied load, the low stress results give more structural stability and model analysis rectangular shaped housings got very less mode frequency in dynamic performances also rectangular shape housings, so rectangular housings suitable for heavy duty applications

\section{REFERENCES}

1. Guruprasad. B, Arun. L, and Mohan. K. "Evaluating For Rear Axle Housing Using Aluminum Composites".

2. Piyush. C. Chaudhari, Vimal. D. Sonara, and Dr.Pravin. P. Rathod. "Analysis and Design of Tractor Rear Axle Using Finite Element Method".

3. A.K. Acharya et al. "Failure Analysis for Rear Axle of Tractor with Loaded Trolley".

4. R. Oyyarvelu, K. Annamalai et al. "Design and Analysis of Front Axle for Two wheel Drive Tractor".

5. Shantanu Ramesh Shinde et al. "Advancement in Simulation of Front Axle of Tractor".

6. Chang-ji Yu, Xue-bing Yu, Modern Design Techniques of Heavy Vehicles Structure, Dalian University of Technology Press, 1998.4. (In China).

7. Wei-xin Liu, Vehicle Design Tsinghua University Press, 2001. (In China).

8. Guo-qiang Wang, Practical Numerical Simulation Technology of Engineering and Practice in ANSYS, Northwest University of Technology Press, 1999.8. (In China).

9. M.M. Topaç a, H. Günal b, N.S. Kuralay, Fatigue failure prediction of a rear axle housing prototype by using finite element analysis, Engineering Failure Analysis, Elsevier, 2008 (Turkey)

10. O.C. Zienkiewicz and R.L. Taylor; The Finite Element Method, McGraw-Hill Co, 1989 (London)

11. C.A. Brebbia, Finite Element Systems, A Handbook; Springer-Verlag; Berlin; 1982.

12. A. Zoo, Reducing the weight of frontal truck axle beam using experimental test procedures to fine tune FEA, Second Worldwide MSC Automotive Conference, Dearborn, Michigan; October 9-11, 2000. 